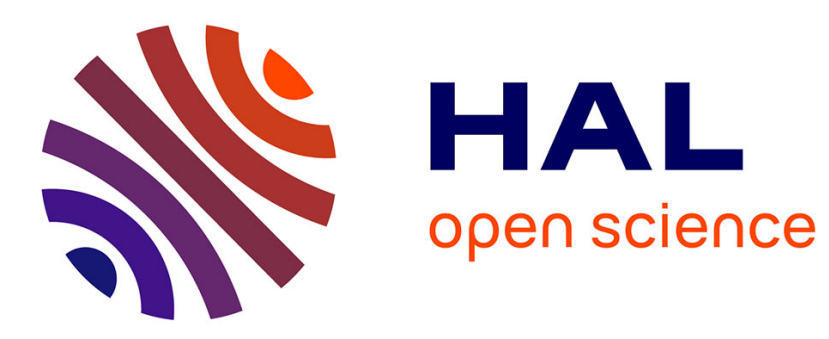

\title{
Zebrafish Models to Study Inflammasome-Mediated Regulation of Hematopoiesis
}

\author{
Lola Rodríguez-Ruiz, Juan Lozano-Gil, Christophe Lachaud, Pablo \\ Mesa-Del-Castillo, María Cayuela, Diana García-Moreno, Ana Pérez-Oliva, \\ Victoriano Mulero
}

\section{To cite this version:}

Lola Rodríguez-Ruiz, Juan Lozano-Gil, Christophe Lachaud, Pablo Mesa-Del-Castillo, María Cayuela, et al.. Zebrafish Models to Study Inflammasome-Mediated Regulation of Hematopoiesis. Current Trends in Immunology, 2020, 10.1016/j.it.2020.10.006 hal-03020978

\section{HAL Id: hal-03020978 https://hal.science/hal-03020978}

Submitted on 24 Nov 2020

HAL is a multi-disciplinary open access archive for the deposit and dissemination of scientific research documents, whether they are published or not. The documents may come from teaching and research institutions in France or abroad, or from public or private research centers.
L'archive ouverte pluridisciplinaire HAL, est destinée au dépôt et à la diffusion de documents scientifiques de niveau recherche, publiés ou non, émanant des établissements d'enseignement et de recherche français ou étrangers, des laboratoires publics ou privés. 
Dear author,

Please note that changes made in the online proofing system will be added to the article before publication but are not reflected in this PDF.

We also ask that this file not be used for submitting corrections. 
1

Q4 Review

\section{Zebrafish Models to Study Inflammasome- - Mediated Regulation of Hematopoiesis}

Q6 Q5 Lola Rodríguez-Ruiz, ${ }^{1}$ Juan M. Lozano-Gil,, ${ }^{1}$ Christophe Lachaud, ${ }^{2}$ Pablo Mesa del Castillo, ${ }^{3}$

6
Hematopoiesis is a complex process through which immature bone marrow precursor cells mature into all types of blood cells. Although the association of hematopoietic lineage bias (including anemia and neutrophilia) with chronic inflammatory diseases has long been appreciated, the causes involved are obscure. Recently, cytosolic multiprotein inflammasome complexes were shown to activate inflammatory and immune responses, and directly regulate hematopoiesis in zebrafish models; this was deemed to occur via cleavage and inactivation of the master erythroid transcription factor GATA1. Herein summarized are the zebrafish models that are currently available to study this unappreciated role of inflammasome-mediated regulation of hematopoiesis. Novel putative therapeutic strategies, for the treatment of hematopoietic alterations associated with chronic inflammatory diseases in humans, are also proposed.

\section{Hematopoietic Lineage Bias in Chronic Inflammation}

Hematopoiesis is the complete process of blood cell formation occurring during embryonic development and adulthood. In vertebrates, this process involves two phases. First, during embryonic development, the primitive hematopoietic wave (see Glossary) is transitory, and the erythroid and myeloid progenitor cells lack pluripotency [1]. In the second phase, the definitive hematopoietic wave $[2,3]$, which occurs at later stages of development, hematopoietic stem cells (HSCs) emerge and give rise to all types of blood cells in adult organisms [4]. HSC maturation involves the differentiation of lymphoid [T, B, and natural killer (NK) cells], myeloid (granulocytes and macrophages), and erythroid (megakaryocytes, erythrocytes) cell lineages [5-7]. Several signaling pathways orchestrate this complex process, by means of different transcription factors regulating the maturation, proliferation, and differentiation of hematopoietic cells. The transcription factors GATA1 and SPI1 (also known as PU.1), are essential and present a cross-inhibitory relationship [8,9] (Figure 1): GATA1 regulates erythroid differentiation [10], and SPI1 regulates myeloid differentiation [11]. However, there are many controversies regarding the factors that may be responsible for terminal erythroid and myeloid differentiation, and the involvement of the HSC niche in these processes [12], given that many unknown pathways seem to play a role in this complex biological process [10,13]. For example, while GATA1 in hematopoietic precursors directs commitment to the erythroid-megakaryocyte lineage [14], the overexpression of GATA1 in erythroid cells inhibits their terminal differentiation in mouse [15]. In addition, GATA1 expression and activity increase in the initial stage of erythroid differentiation, but they both decrease in later stages to allow terminal erythroid differentiation in mouse and human [16]. All these complex pathways might have important clinical implications, since hematopoietic lineage bias, mainly neutrophilia and anemia, are associated with an increased incidence of disease, at least for 30 pathologies with prominent inflammatory components, including infection, rheumatoid arthritis, atherosclerosis, inflammatory bowel diseases, kidney and pulmonary diseases, autoimmune conditions, neurodegenerative diseases, and cancer [17]. In this review, the general structure and function of mammalian inflammasomes, and the specificities of macrophage and neutrophil inflammasomes (which are the best characterized), are summarized. Then follows a discussion on

\section{Highlights}

The activation of the Nlrp1a inflammasome in mouse hematopoietic stem and progenitor cells (HSPCs) triggers their pyroptotic death and prolonged leukopenia upon chemotherapy or viral infection.

The NLRP3 inflammasome drives HSPC clonal expansion and pyroptosis in myelodysplastic syndrome patients.

Emerging evidence from zebrafish models suggests that the canonical inflammasome autonomously regulates the erythroid-myeloid cell fate decision of HSPCs, and terminal erythroid differentiation through the cleavage of Gata1. This mechanism appears to be evolutionarily conserved since pharmacological inhibition of caspase 1 (Casp1) in mouse hematopoietic stem cells (HSCs) results in a rapid increase in Gata1 protein, leading to erythroid differentiation.

Pharmacological inhibition of the canonical inflammasome rescues zebrafish disease models of neutrophilic inflammation, anemia associated with chronic diseases, and Diamond-Blackfan anemia.

${ }^{1}$ Departamento de Biología Celular e Histología, Facultad de Biología, Universidad de Murcia, IMIB-Arrixaca, Centro de Investigación Biomédica en Red de Enfermedades Raras, 30100 Murcia, Spain

${ }^{2}$ Aix-Marseille Univ, INSERM, CNRS, Institut Paoli-Calmettes, CRCM, Marseille, France

${ }^{3}$ Hospital Clínico Universitario Virgen de la Arrixaca, IMIB-Arrixaca, Centro de Investigación Biomédica en Red de Enfermedades Raras, 30120 Murcia, Spain 


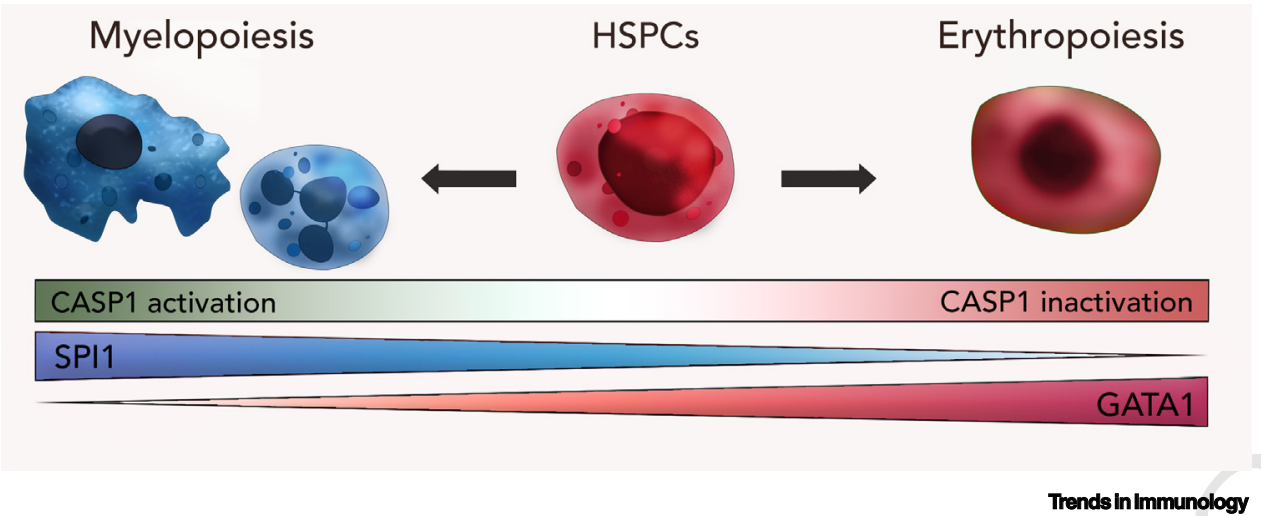

Figure 1. Regulation of HSPC Lineage Decision Choice by the Canonical Inflammasome in Zebrafish and Mouse. Activation of the inflammasome in HSPCs results in the cleavage and inactivation of GATA1, promoting increased amounts of SPI1 that promotes myeloid differentiation. Conversely, inhibition of the inflammasome in HSPCs results in an increased GATA1 amount that promotes erythroid differentiation [30]. Abbreviation: CASP1, caspase 1; HSPCs, hematopoietic stem and progenitor cells.

the main advantages of using the zebrafish to model inflammation and inflammasomes, describing how this model has highlighted the importance of inflammasomes in hematopoiesis. Indeed, this model may help explain the hematopoietic lineage bias that has been associated with chronic inflammation in humans, revealing novel candidate therapeutic targets for future investigation in some of these diseases. (See Box 1.)

\section{Inflammasomes}

Inflammasomes act as sensors of the innate immune system in vertebrates, and promote inflammation in response to various stimuli, including infection, tissue damage, or metabolic dysregulation. They are multiprotein complexes formed by a receptor, the adaptor protein apoptosis-associated speck-like protein containing a CARD (ASC), and an effector that interacts through homotypic interactions [18]. Recently, the guanylate-binding protein (GBP) family was found to also form a part of these multiprotein complexes in humans, mice, and zebrafish [19-22]. Inflammasomes act by activating inflammatory caspases, namely caspase 1 (CASP1) (canonical inflammasomes), and CASP4, CASP5 (human), and Casp11 (mouse) (noncanonical inflammasomes) [18].

Inflammasome sensors are grouped, depending on their structure, into nucleotide-binding domain (NOD)-like receptors (NLRs), absent in melanoma 2 (AIM2)-like receptors (ALRs), or pyrin [18]. When a specific stimulus is detected, the sensor interacts with and promotes the oligomerization of ASC, which forms specks and is able to recruit and activate inflammatory

\section{Box 1. Anemia-Associated with FMF and CAPS}

Familiar Mediterranean Fever (FMF) and cryopyrin-associated periodic syndrome (CAPS) are two inflammatory diseases driven by germ-line mutations in genes encoding inflammasome components. FMF is the most common monogenic autoinflammatory disease worldwide, affecting an estimated 150000 patients [88]. More than $60 \%$ of FMF patients develop anemia that is associated with iron status rather than inflammation, assayed as plasma IL-6 concentrations [89]. Similarly, iron-resistant hypochromic anemia has also been associated with CAPS [90]. It is tempting to speculate that overactivation of the inflammasome in HSPCs could directly promote anemia by enforcing erythropoiesis, explaining the usual iron-refractory nature of these diseases, but this remains to be robustly investigated. Furthermore, released Asc specks are able to propagate inflammation in mouse models of CAPS and are found in the serum of patients with CAPS, but not with FMF $[60,61]$. Therefore, macrophage-derived ASC specks may be taken up by HSPCs, activating their inflammasome and further enhancing myelopoiesis at the expense of erythropoiesis, which might explain the ironresistance anemia-associated with CAPS (see Clinician's Corner and Outstanding Questions) (Figure 2).

*Correspondence: dianagm@um.es (D. García-Moreno), anabpo@um.es (A.B. Pérez-Oliva), and vmulero@um.es (V. Mulero). 
caspases. For a long time, CASP1 autoproteolysis was deemed to be triggered by the proximity of its active subunits p20 and p10 [23]; however, this view was recently challenged in an elegant study [24]. The authors demonstrated in mouse models that the dominant species of active CASP1 dimers, were in fact full-length p46 dimers and a transient species, p33/p10. In addition, p33/p10 autoprocessing occurred with kinetics specified by the inflammasome size and the cell type, resulting in the release of $\mathrm{p} 20 / \mathrm{p} 10$ species, which became unstable, and protease activity was terminated [24]. Therefore, the inflammasome also incorporates an intrinsic self-limiting mechanism that ensures timely CASP1 deactivation [24].

Caspase dimerization and auto-proteolytic maturation lead to the processing and secretion

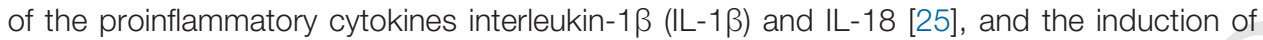
a form of programmed cell death called pyroptosis [18]. Pyroptosis leads to the loss of membrane integrity, which in turn, mediates the release of intracellular contents into the extracellular fluid [26]. Both IL-1ß/18 release and pyroptosis are mediated by Gasdermin D (GSDMD) $[27,28]$.

In addition to its role in infection and inflammation, inflammasomes are also important in maintaining basic cellular functions, such as autophagy, metabolism, cellular proliferation, gene transcription, and tumorigenesis [29]. Recent findings have also indicated that inflammasomes play an essential role in the regulation of hematopoiesis in zebrafish, mouse, and human [30,31,32-35]. This is relevant, as uncontrolled inflammasome activation has led to certain human autoimmune and metabolic disorders, underscoring the importance of gaining a better understanding of this process in physiological and pathological contexts [30].

\section{Cell Type-Specific Inflammasomes}

Neutrophils and monocytes/macrophages belong to the myeloid lineage originating from a common granulocyte-monocyte progenitor (GMP), which arises from the differentiation of HSCs [7]. Neutrophils are the most abundant circulating leukocytes (50-70\%) in most mammals, released from the bone marrow (BM) upon CXCL8, CXCL1, and CXCL2 chemokine signaling [36]. In a similar way, monocytes are released from the BM upon CCL2 and CCL7 signaling, via CCR2 [37]. Subsequently, they are extravasated by signals emanating from the activated endothelium close to inflamed tissues [38-40]. The production of neutrophils increases during infection or inflammation in a process called 'danger mobilization' or 'emergency hematopoiesis', mainly triggered by the hematopoietic cytokine granulocyte colony-stimulating factor (G-CSF) [41].

Neutrophil and macrophage inflammasomes sense infection and sterile inflammation, through canonical and noncanonical pathways $[39,42,43]$. Both cell types express primarily a wide range of NLRs and ALRs, and are the first cells to participate in the inflammatory process [44] (Table 1). Nonetheless, there are differences between inflammasomes in these cells, enabling the complex and specific regulation of cellular processes. Although they show similar amounts of NOD-, LRR-, and pyrin domain-containing protein 3 (NLRP3) and NOD-, LRR-, and CARD domain-containing protein 4 (NLRC4), the expression of NLRP12 is higher in mouse neutrophils than in macrophages [45]. The activation of NLRC4 in human macrophages occurs through specific Gram-negative bacteria components $[46,47]$. Pyroptosis is induced in macrophages by ASC-independent NLRC4 signaling, or ASC-dependent NLRP3 and AIM2 inflammasome activation, facilitating the elimination of intracellular pathogens [48]. Even though NLRC4 or AIM2 signaling has also been observed in human and mouse neutrophils, they do not seem to induce pyroptosis [45]. Thus, neutrophils are quite resistant to pyroptosis and intracellular infection by bacteria [45]. Accordingly, they also engage in sustained release of IL-1B, thus amplifying neutrophil recruitment and activation [45]. Furthermore, when human neutrophil inflammasomes are activated by canonical agonists, IL-1B,

\section{Glossary}

\section{Activated endothelium:}

proinflammatory and procoagulant state of endothelial cells lining the lumen of blood vessels allowing extravasation of leukocytes.

AIM2-like receptors (ALRs): intracellular inflammasome receptors detecting the presence of DNA in cytosol.

Apoptosis-associated speck-like protein containing a CARD (ASC): key adaptor protein required to activate the inflammasome.

BM hypoplasia: type of hypoplastic anemia.

Canonical inflammasome: effector is CASP1 (mammals) or Caspa (zebrafish). Caspase a (Caspa): functional homolog of CASP1 in zebrafish. Caspase 1 (CASP1): inflammatory caspase involved in processing $\mathrm{IL}-1 \beta$, IL-18, and GSDMD.

Caspases: family of cysteine proteases that cleave after aspartate residues (Asp).

Chronic obstructive pulmonary disease (COPD): type of obstructive lung disease characterized by long-term breathing problems and poor airflow. Complement activation: the initiation of a series of reactions involving the complement proteins of plasma that may result in either the elimination of the pathogenic organism or amplification of the complement cascade effect. Cryopyrin-associated periodic syndrome (CAPS): group of rare autoinflammatory diseases characterized by IL-1 $\beta$-mediated systemic inflammation; caused by gain-of-function mutations in the NLRP3 gene.

CRISPR-Cas technology: a genome editing technique used to modify the genomes of living organisms. Definitive hematopoietic wave: the latter of two waves of hematopoiesis where HSPCs are generated. Diamond-Blackfan anemia: congenital anemia caused by inefficient translation of GATA1.

Familial Mediterranean fever (FMF): autoinflammatory disease caused by mutations in the MEFV gene, encoding pyrin.

Gasdermin D (GSDMD): substrate of inflammatory caspases; mediates pyroptosis.

GATA1: transcription factor regulating the expression of genes involved in erythropoiesis and thrombopoiesis. 
but not IL-1 $\alpha$ and IL-18, is released, indicating that IL-1 $\beta$ and IL-18 protein release is differentially regulated in human neutrophils [42].

The NLRP3 inflammasome is involved in inflammatory and metabolic disorders as well as host defense [44]. Although, it is also present in neutrophils, it acts differently. NLPR3 signaling in neutrophils is only activated through soluble agonists, but it can be triggered by soluble and particulate, or crystalline agonists in mouse macrophages [49]. In addition, NLRP3 activation leads to different cellular outcomes in macrophages and neutrophils. Both NLRP3/CASP1-dependent and NLRP3/CASP8-dependent inflammasomes lead to pyroptosis and apoptotic cell death in mouse macrophages, respectively, but not in neutrophils [50-52].

Regarding hematopoietic stem and progenitor cells (HSPCs), the expression/activation of inflammasomes has received little attention. Nevertheless, the genes encoding the main components of the NLRP3 and NLRP1 inflammasome, including NLRP3, ASC, and CASP1, are expressed in human and mouse HSPCs [31,34]. Similarly, nascent HSPCs in zebrafish embryos have been reported to express Asc [53]. Therefore, each cell type shows different inflammasome components, activation mechanisms, and effector functions.

\section{Inflammasomes in Diseases}

Numerous data from mouse models, supported by human clinical data, strongly support the role of the inflammasome in the initiation and/or progression of diseases, with a strong impact on public health; these include certain metabolic disorders and neurodegenerative diseases [54]. However, the strongest evidence supporting a key role for inflammasomes in human pathology comes from numerous genetic association studies in a variety of diseases [55], consistent with their importance in the regulation of inflammation and tissue repair. The most convincing evidence comes from point mutations in a coding amino acid of the inflammasome sensors MEFV (coding for pyrin), NLRP3, and NLRP1, which lead to familial Mediterranean fever (FMF), cryopyrin-associated periodic syndrome (CAPS), and skin inflammatory and cancer susceptibility syndromes, respectively, highlighting the clinical significance of these proteins [54,56-59]. Furthermore, two recent studies demonstrated the presence of ASC specks in the serum of patients with chronic inflammatory disorders such as chronic obstructive pulmonary disease (COPD) and CAPS, and these might be considered as biomarkers of active disease $[60,61]$. It is speculated that extracellular specks might also contribute to amplifying inflammation, as well as neutrophilia and anemia in human chronic inflammatory disorders such as these; it is possible that they might do so by altering the erythroid-myeloid fate decision of HSPCs [31] when reaching the BM, but this remains to be demonstrated (see Clinician's Corner) (Figure 2, Key Figure).

From another angle, the presence of neutrophils in atherosclerotic plaques correlates with the instability of these plaques, and a poor prognosis in atherosclerosis; this instability has also been associated with the formation of neutrophil extracellular traps (NETs) and inflammasome activation, since NETs have been detected in atherosclerotic lesions and arterial thrombi in humans and mice, and can induce the activation of endothelial cells, antigen-presenting cells, and platelets $[62,63]$. Moreover, both types of cellular death, pyroptosis and NETosis, need GSDMD cleavage; however, while mouse neutrophils use inflammasome-dependent mechanisms to cleave GSDMD and activate NETosis, human neutrophils use neutrophil elastase to do so [64,65]. Altogether, these observations suggest that chronic activation of macrophage and neutrophil inflammasomes can disrupt homeostasis in an organism and potentially contribute to inflammatory diseases; as such, inflammasomes might represent promising therapeutic targets for restoring homeostasis in a variety of pathologies, but this evidently remains to be further investigated.
Guanylate-binding proteins (GBP): family of GTPases induced by IFN-y and regulating inflammasome activation. Granulocyte colony-stimulating factor (G-CSF): glycoprotein that stimulates the BM to produce granulocytes and stem cells, releasing them into the bloodstream Hematopoietic stem and progenitor cells (HSPCs): cells from which differentiated blood cells originate. HSPC niche: anatomical location in which HSPCs reside and self-renew. Inflammasomes: cytosolic

multiprotein oligomers of the innate immune system responsible for the induction of inflammation. Leukopenia: low white blood cell count.

Morpholino: a DNA oligonucleotide used to modify gene expression through blocking mRNA splicing or translation.

Myelodysplastic syndrome: group of cancers in which immature blood cells in the bone marrow do not mature. Myeloid: cells including neutrophils, monocytes, macrophages, myeloid dendritic cells, and mast cells. Myeloperoxidase: peroxidase abundant in neutrophil lysosomes. Neutrophil extracellular traps (NETs): network of extracellular fibers composed primarily of DNA from neutrophils that bind pathogens and allow their clearance.

Neutrophilia: high number of neutrophils in the blood.

Neutrophilic dermatosis: group of inflammatory skin disorders comprising sterile inflammation and neutrophilic skin infiltration.

NOD-like receptors (NLRs): inflammasome receptors detecting pathogens and cytosolic signals of cell stress or tissue damage.

Noncanonical inflammasome: effector is human CASP4 and CASP5, or murine Casp11.

Pluripotency: capacity to self-renew by dividing and developing into the three primary germ cell layers of the early embryo and therefore, into all cells of the adult.

Primitive hematopoietic wave: the first of the two waves of hematopoiesis. It is transitory and gives rise to erythrocytes and myeloid cells (macrophages and neutrophils) during early embryonic development. PYRIN: inflammasome receptor sensing modifications in the activity of the small GTPase RhoA 
The Zebrafish: A Unique Model for Studying Hematopoiesis and Inflammation The zebrafish has become an exceptional model organism in many fields of biomedical research. It is the only vertebrate model that has the main advantages of invertebrates (e.g., the fruit fly and Caenorhabditis elegans), including: (i) high fecundity (between 200 and 300 eggs per female can be obtained each week), and the embryonic development is rapid and external; (ii) the embryos are transparent, allowing the development and tracking of their cells in tissues and organs using optical and fluorescent methods; and (iii) the animal is suitable for high throughput chemical and genetic screenings [66]. In addition, its genome is completely sequenced and shares $70 \%$ of its genes with humans, where up to $82 \%$ of them are known to be relevant/causative of human diseases [67]. This high genetic homology allows transgenesis studies with human genes and enables the in vivo study of gene regulation and function.

Zebrafish have been used as a research model to study hematopoiesis [68]. Although hematopoiesis in zebrafish occurs in sites that are distinct from other vertebrates (e.g., zebrafish HSCs reside in the kidney marrow), the genetic programs controlling hematopoiesis are conserved
Pyroptosis: highly inflammatory form of programmed cell death promoted by inflammasomes.

Specks: large protein complex of ASC formed after inflammasome activation. SPI1: transcription factor regulating the expression of genes involved in myelopoiesis and B lymphocyte development.

Transcription activator-like effector nucleases (TALENs): restriction enzymes that can be engineered to cut specific sequences of DNA.

Table 1. Components and Effector Functions of the Main Canonical Inflammasomes in HSPCs, Erythroid Progenitors, and Mature Myeloid Cells (Macrophages and Neutrophils) in the Indicated Species ${ }^{\mathrm{a}, \mathrm{b}}$

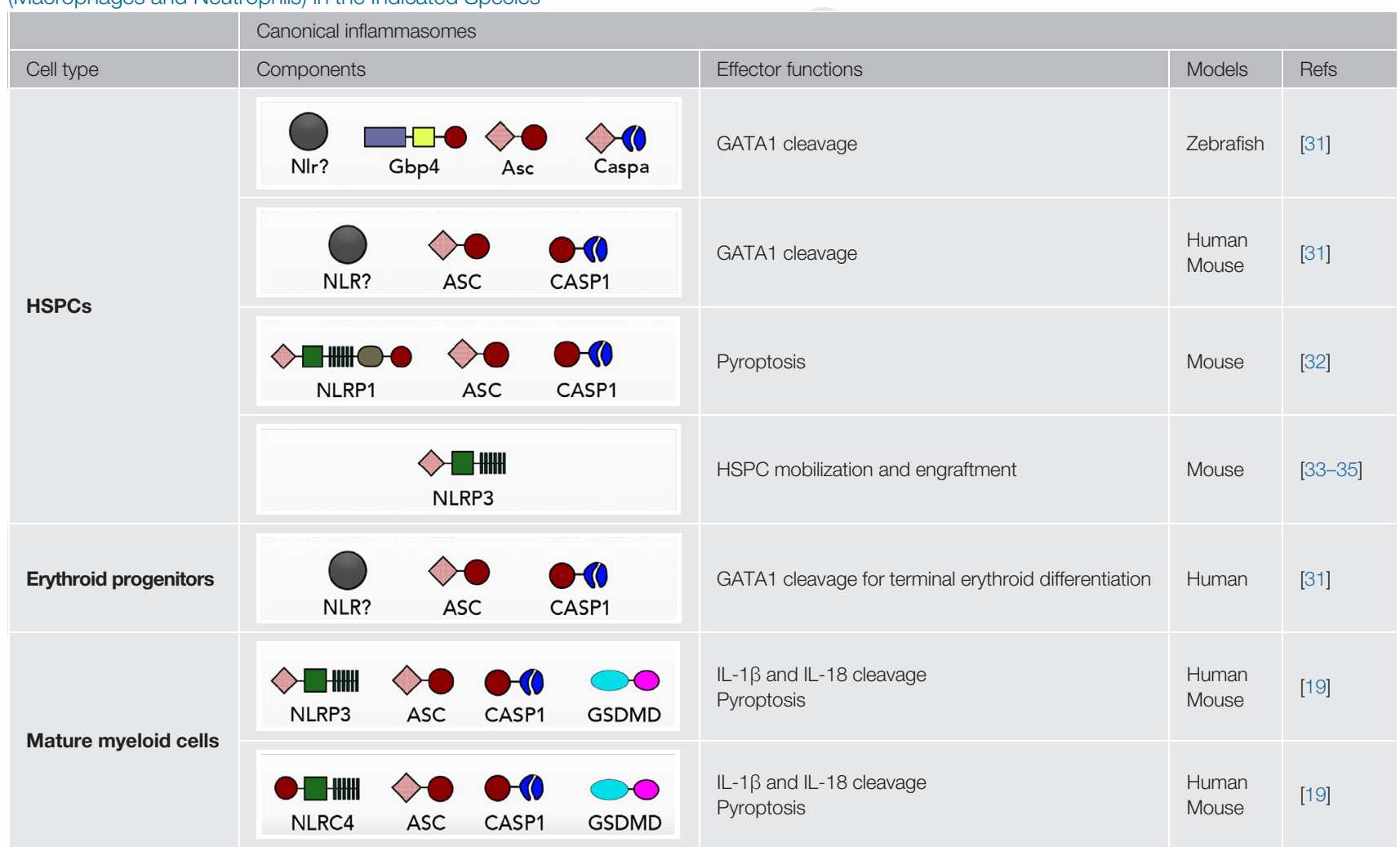

Unknown sensor

PYD domain

NACHT domain

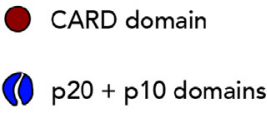

IIIIII LRR domains

a Abbreviations: ASC, apoptosis-associated speck-like protein containing a CARD; CARD, caspase activation and recruitment domains; CASP1, caspase 1; GBP, guanylatebinding proteins; GSDMD, Gasdermin D; HSPCs, hematopoietic stem and progenitor cells; NLRs, nucleotide-binding domain-like receptors; PYD, pyrin domain.

${ }^{\mathrm{b}}$ The domains of each inflammasome component are shown. 


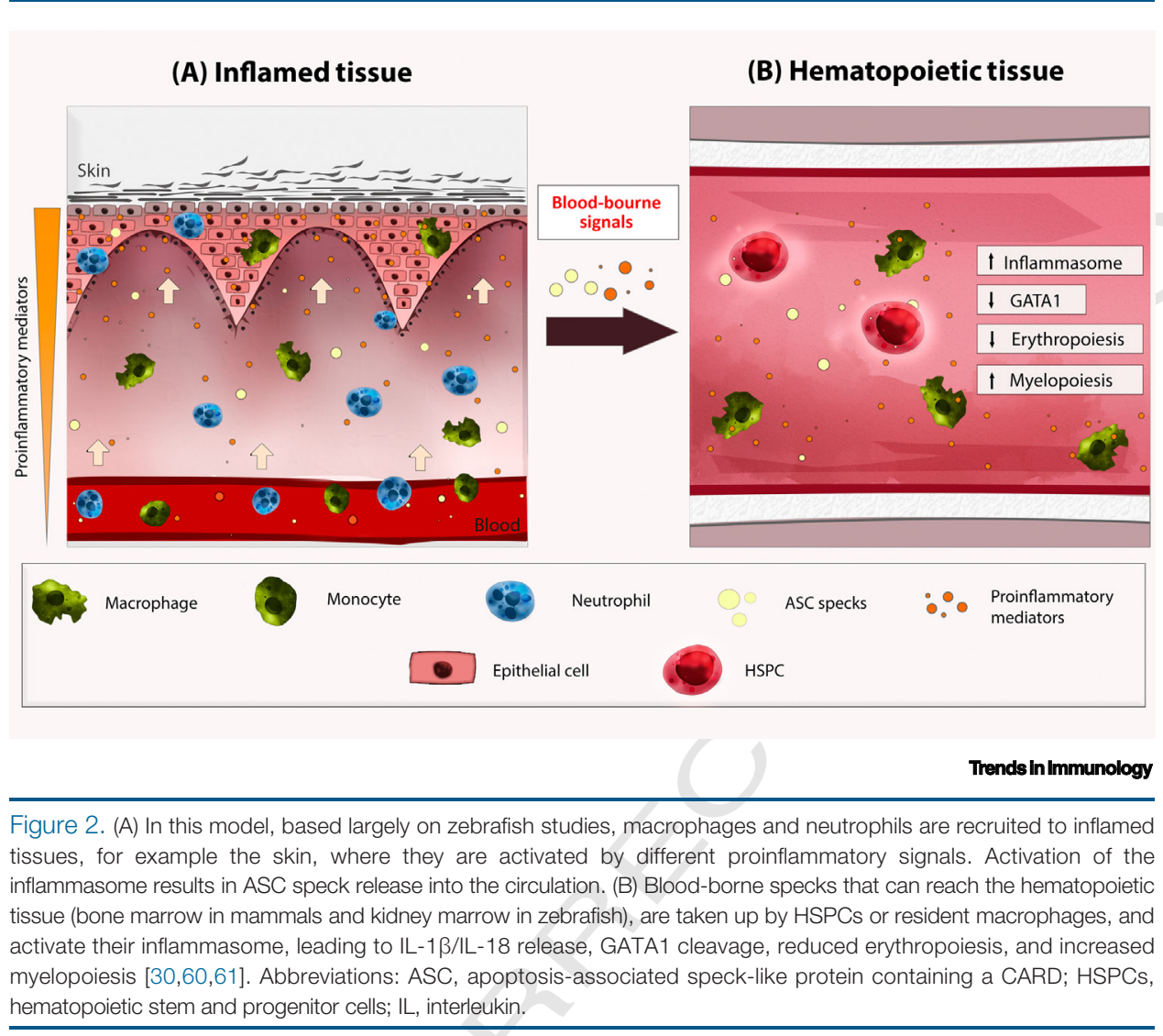

between zebrafish and mammals, including humans, making zebrafish a clinically relevant model system [4]. The earlier mentioned advantages, together with the construction of detailed genomic databases, have allowed a series of genetic approaches to be used in this model for the study of a variety of blood disorders, such as congenital and acquired anemia, neutrophilia, and leukemia [69]. Zebrafish has also consolidated its position as a powerful animal model in the fields of immunity, inflammation, and infection [70,71]. It has contributed to improving our knowledge of human infections, such as tuberculosis which is difficult to model in vivo in mammals [72]. In addition, the optical transparency of the early life stages of zebrafish offers unprecedented possibilities for studying the activation and dynamics of inflammasomes in vivo [73].

Gain (mRNA) and loss of function experiments in embryos [morpholinos, transcription simple to perform, enabling the study of the role of specific molecules, including those involved in the inflammasome, and these roles have been better deciphered using different stimuli and pathogens [71]. In addition, zebrafish has allowed the analysis and visualization of different types of immune cells, including neutrophils and macrophages; indeed, the assembly of inflammasomes has been studied using transgenic zebrafish lines that are either already available, 
or easy to generate [74]. Among the numerous transgenic lines available to study hematopoiesis and inflammasomes, the following should be mentioned (Figure 3): $\operatorname{Tg}(m p x$ :eGFP) [75] and Tg(lyz: dsRed) [76], which express fluorescent eGFP or dsRed driven by the neutrophil specific

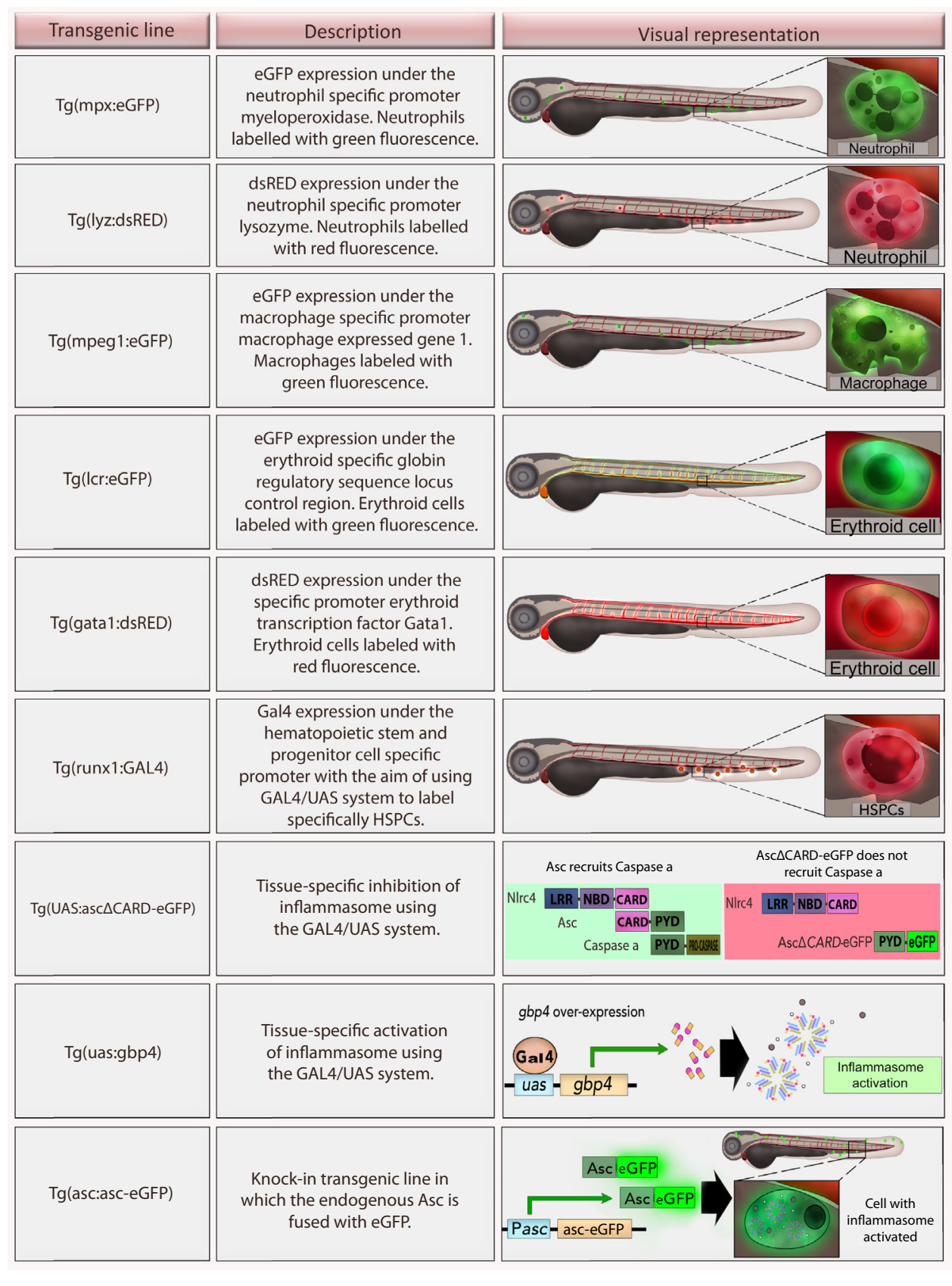

Trends in Immunology

Figure 3. Zebrafish Transgenic Lines in the Study of Inflammation and the Inflammasome in Hematopoiesis. Specific hematopoietic cell types and the visualization of the inflammasome assembly in real time have been feasible using these lines $[30,73]$. Chemical and genetic screening methods are also amenable in zebrafish, sharing certain advantages of invertebrate models for high throughput screening and in vivo imaging, such as high fecundity, optical transparency, and rapid and external development. Abbreviations: ASC, apoptosis-associated speck-like protein containing a CARD; HSPCs, hematopoietic stem and progenitor cells; PYD, pyrin domain. 
promoters myeloperoxidase and lysozyme, respectively; Tg(mpeg1:eGFP) [77], with labeled macrophages stemming from activation of the mpeg1 promoter; and Tg(Icr:eGFP) [78] which has green fluorescent erythrocytes due to the expression of eGFP, driven by a globin regulatory sequence. Moreover, another two lines can be used to study erythropoiesis: Tg(gata1:DsRed) [79], which allows visualization of erythroid cells because they express dsRed driven by the gata1 promoter; and Tg(runx1:GAL4) [80] with its labeled HSPCs. Finally, the knock-in line Tg (asc:asc-eGFP) allows real-time single cell visualization of inflammasome assembly [31], and the lines $T g$ (uas:asc $\triangle$ CARD-eGFP) and Tg(uas:gpb4) allow the respective inhibition or activation of the inflammasome in specific cell types [64].

\section{The Zebrafish Model Sheds Light on the Importance of the Inflammasome in Hematopoiesis}

As mentioned, a relationship between inflammasome activation and hematopoiesis has been suggested for several medical conditions; for instance, hematopoietic lineage bias has been associated with chronic inflammatory diseases. However, the mechanisms and signaling pathways involved are unknown. One of the first pieces of evidence for this was the demonstration that human GATA1 could be cleaved in vitro by many caspases, and in vivo by caspase- 3 in human erythroid precursors $[81,82]$. Furthermore, the activation of NIrp1a in mouse HSPCs triggers pyroptotic death of these cells and leukopenia in the absence of inflammation, while in periods of hematopoietic stress induced by chemotherapy or viral infection, NLRP1a activation caused prolonged cytopenia, BM hypoplasia, and immunosuppression [31]. In addition, the

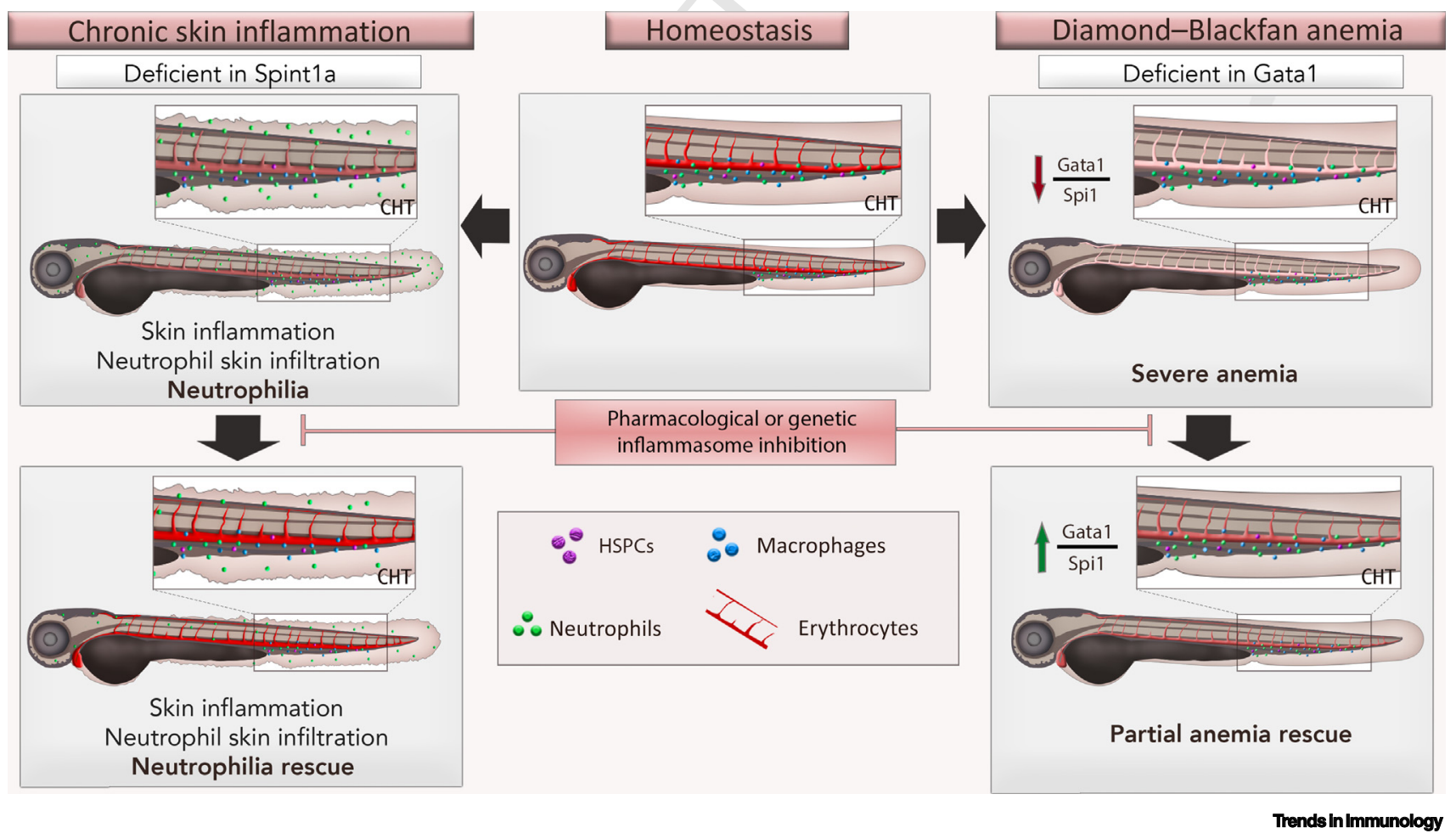

Figure 4. Zebrafish Disease Models for Studying Hematopoiesis. In homeostasis conditions, embryonic hematopoiesis takes place in the caudal hematopoietic tissue (CHT) 2-3 days postfertilization (middle panel). The Spint1a-deficient line (spint1 $\mathrm{a}^{\text {hiz217Tghiz217Tg }}$ is an excellent model for studying hematopoietic lineage bias associated with chronic inflammation, since it leads to neutrophilia which can be visualized (left panel). The Gata1 amorphant animal is an amenable model of Diamond-Blackfan anemia. In both models, inhibition of the inflammasome can rescue hematopoietic lineage bias [30]. Abbreviation: HSPCs, hematopoietic stem and progenitor cells. 
NLRP3 inflammasome is required for the optimal release of mouse HSPCs from the BM in response to purinergic signaling, complement activation, and G-CSF administration [32,33]. Notably, transplantation experiments in mouse have shown that NLRP3 knockout, either in HSPCs or in BM stromal cells, results in impaired HSPC homing and engraftment [34]. Furthermore, the NLRP3 inflammasome has been found to drive clonal expansion and pyroptotic cell death in myelodysplastic syndrome patients [35] (see Clinician's Corner). Accordingly, it has been demonstrated that, while the canonical inflammasome is dispensable for HSPC emergence in zebrafish larvae, it autonomously regulates HSPC differentiation in homeostatic conditions at two different levels: erythroid-myeloid cell fate decision and terminal erythroid differentiation [30] (Figure 1 and Table 1). Thus, the genetic or chemical inhibition of different inflammasome components, \{e.g., Gbp4 [Tg(uas:gpb4)], Asc Tg(uas:asc $\triangle$ CARD-eGFP)], or caspase a (Caspa)\}, (the zebrafish functional equivalent of pharmacological inhibition of mammalian CASP1), resulted in a decreased number of neutrophils and macrophages accompanied by a concomitant increase in erythrocyte counts in larvae, while conversely, the forced expression of both Asc and Caspa simultaneously by mRNA injection, increased neutrophil numbers [30]. These results were further confirmed by neutrophil ablation experiments, achieved by treating larvae, which express the bacterial nitroreductase in neutrophils with the prodrug metronidazole; pharmacological inhibition of CASP1 fully impaired neutrophil recovery after ablation. Further analysis showed that the emergence of HSPCs in Tg(runx1:GAL4) was not affected, suggesting that the inflammasome could regulate HSPC differentiation [30]. Mechanistically, inflammasome activation in HSPCs resulted in reduced Gata1 protein amounts, enforcing myelopoiesis and inhibiting erythropoiesis. The mechanism seems to be evolutionarily conserved, since the pharmacological inhibition of CASP1 in HSCs from SPI1-eYFP and GATA1-mCherry reporter mice rapidly increased Gata1 protein amounts, without affecting those of Spi1, resulting in increased erythroid differentiation at the expense of myeloid differentiation (Figure 1) [30]. In addition, erythroid differentiation of the human erythroleukemic line K562 was also impaired upon inhibition of CASP1, but not CASP4, suggesting a role for the canonical inflammasome in terminal erythroid differentiation, by dampening GATA1 protein amounts [30]. In vitro experiments confirmed the ability of human recombinant CASP1 to cleave GATA1 at residue D300 [30]. Taken together, these results support a scenario in which GATA1 protein amounts are fine-tuned by the canonical inflammasome in HSPCs, to regulate the erythroid-myeloid decision, and simultaneously in erythroid progenitors, to regulate their terminal differentiation. Furthermore, the inflammasome and macrophage-derived IL-1 $\beta$ appear to be required for the expansion of HSPCs of zebrafish embryos, assayed by whole-mount in situ hybridization of the HSPC markers runx $1 / \mathrm{cmyb}$ and Tg (cd41:eGFP) larvae [58]. Further studies are required, however, to elucidate the signals responsible for activating the inflammasome in HSPCs in homeostasis, as well as the inflammasome components involved (see Outstanding Questions).

This close relationship between GATA1 and CASP1 is relevant at a clinical level, since bias of the hematopoietic lineage has been associated with a higher incidence of diseases with an inflammatory component, such as infection, rheumatoid arthritis, atherosclerosis, inflammatory bowel diseases, kidney and pulmonary diseases, autoimmune pathologies, neurodegenerative diseases, and certain cancers [17]. An example of this group of diseases is neutrophilic dermatosis, characterized by the accumulation of neutrophils in polymorphic cutaneous lesions of patients [83]. A zebrafish line deficient in the serine peptidase inhibitor, Kunitz type 1a (Spint1a), has been used as a model of this disease, since it shows neutrophilia, skin lesions, and high skin neutrophil infiltration [84,85]. Accordingly, inhibition of the canonical inflammasome with the CASP1 inhibitor Ac-YVAD-CMK in this model, restored Gata1 amounts and decreased the number of neutrophils, even though both skin lesions and neutrophil infiltration were not affected [30] (Figure 4).

\section{Clinician's Corner}

Alterations in inflammasome activity might have important clinical implications, since hematopoietic lineage bias has been associated with certain chronic diseases with prominent inflammatory components, including infections, rheumatoid arthritis, atherosclerosis, inflammatory bowel diseases, kidney and pulmonary diseases, autoimmune conditions, neurodegenerative diseases, and cancer $[17,83,91]$.

Emerging evidence suggests that the inflammasome may play a key role in a variety of human diseases, including FMF, CAPS, and skin inflammatory and cancer syndromes, which are driven by gain-of-function mutations in MEFV, NLRP3, and NLRP1, respectively [54,56-59]. As many FMF and CAPS patients develop iron-resistant hypochromic anemia, inflammasome overactivation in HSPCs might contribute to anemia, which might explain its iron-refractory nature, although this warrants further investigation.

NLRP3 and ASC specks are present in the serum of CAPS and COPD patients, and might be considered as putative biomarkers of active disease $[60,61]$. These specks might contribute to amplifying not only inflammation, but also neutrophilia and anemia, after being taken up by HSPCs, activating their inflammasome, and promoting hematopoietic lineage bias through GATA1 cleavage and inactivation. This also requires further testing. 
Another disease in which inhibition of the canonical inflammasome might be considered a putative therapeutic target for further exploration is Diamond-Blackfan anemia (DBA), a hereditary disease in which erythropoiesis is impaired due to reduced GATA1 protein amounts [86]. We modeled DBA in zebrafish by reducing Gata1 amounts using a specific morpholino [87]. Pharmacological inhibition of Caspa with Ac-YVAD-CHO in this DBA model was able to partially rescue anemia and to increase Gata1 and to decrease Spi1 protein amounts [30] (Figure 4). Taken together, pharmacological inhibition of the inflammasome rescued neutrophilic inflammation and anemia in these zebrafish disease models [30]; this suggested that the inflammasome can play a major role in hematopoietic lineage bias during certain chronic inflammatory diseases and DBA, at least stemming from zebrafish models. Nevertheless, these findings certainly merit further attention in identifying novel druggable targets for potential therapeutic interventions in some of these conditions (see Clinician's Corner).

\section{Concluding Remarks}

The zebrafish model has unique advantages for biomedical research and, herein discussed is its relevance for designing studies to better understand the roles and mechanisms of inflammasome activation in hematopoiesis. The availability of models of chronic inflammatory diseases, including neutrophilic dermatosis and rare diseases such as DBA, provides an excellent and unique tool to rapidly identify novel treatments for some of these pathologies, and furthermore, for screening clinically approved drug libraries that may facilitate relatively rapid repurposing. In addition, powerful CRISPR-Cas9 technology allows the rapid generation of candidate pathogenic mutations, which can even be analyzed in the FO generation without the need for generating timeconsuming deficient lines, to confirm their pathogenicity, and target or lead drug screening (see Clinician's Corner). Finally, it is essential to find the specific inflammasome(s) involved in hematopoiesis to develop more specific treatments for the hematopoietic lineage bias associated with chronic inflammatory disorders (see Outstanding Questions). The possibility of in vivo tracking of hematopoietic cells, together with the inflammasome tools available, including reporters to visualize its assembly in real time, can make this model complementary to other models of vertebrate species, to study the crosstalk between hematopoiesis and inflammation in general, and the inflammasome in particular.

\section{Acknowledgments}

The work in our laboratory is funded by the Spanish Ministry of Science and Innovation (grant BIO2017-84702-R to V.M.) and PhD fellowship to L.R-R., both cofunded with Fondos Europeos de Desarrollo Regional/European Regional Development Funds, ISCIII-CIBERER (grant CB19/07/00031 to V.M.), Fundación Séneca, Agencia de Ciencia y Tecnología de la Región de Murcia (grant 20793/PI/18 to V.M.), and the University of Murcia (postdoctoral contract to D.G-M.).

\section{References}

1. Palis, J. and Yoder, M.C. (2001) Yolk-sac hematopoiesis: the first blood cells of mouse and man. Exp. Hematol. 29, 927-936

2. McGrath, K.E. et al. (2011) A transient definitive erythroid lineage with unique regulation of the $\beta$-globin locus in the mammalian embryo. Blood 117, 4600-4608

3. Bertrand, J.Y. et al. (2007) Definitive hematopoiesis initiates through a committed erythromyeloid progenitor in the zebrafish embryo. Development 134, 4147-4156

4. Jagannathan-Bogdan, M. and Zon, L.I. (2013) Hematopoiesis. Development 140, 2463-2467

5. Weissman, I.L. (2000) Translating stem and progenitor cell biology to the clinic: barriers and opportunities. Science 287 1442-1446

6. Kondo, M. et al. (2003) Biology of hematopoietic stem cells and progenitors: implications for clinical application. Annu. Rev. Immunol. 21, 759-806

7. Kondo, M. (2010) Lymphoid and myeloid lineage commitment in multipotent hematopoietic progenitors. Immunol. Rev. 238, $37-46$
8. Zhang, P. et al. (2000) PU.1 inhibits GATA-1 function and 343 erythroid differentiation by blocking GATA-1 DNA binding. 344 Blood 96, 2641-2648 345

9. Rekhtman, N. et al. (1999) Direct interaction of hematopoietic 346 transcription factors PU.1 and GATA-1: functional antagonism 347 in erythroid cells. Genes Dev. 13, 1398-1411 348

10. Cantor, A.B. and Orkin, S.H. (2002) Transcriptional regulation of 349 erythropoiesis: an affair involving multiple partners. Oncogene 350 21, 3368-3376 351

11. Scott, E.W. et al. (1994) Requirement of transcription factor PU.1 352 in the development of multiple hematopoietic lineages. Science 353 265, 1573-1577 354

12. Wattrus, S.J. and Zon, L.I. (2018) Stem cell safe harbor: the hema- 355 topoietic stem cell niche in zebrafish. Blood Adv. 2, 3063-3069 356

13. Hoppe, P.S. et al. (2016) Early myeloid lineage choice is not initiated 357 by random PU.1 to GATA1 protein ratios. Nature 535, 299-302 358

14. Iwasaki, H. et al. (2003) GATA-1 converts lymphoid and 359 myelomonocytic progenitors into the megakaryocyte/erythrocyte 360 lineages. Immunity 19, 451-462 361

\section{Outstanding Questions}

What signals are responsible for activating the inflammasome in HSPCs? What is the cellular source of these signals - do they have a stromal or hematopoietic origin?

Are the signals activating the HSPC inflammasome the same in steady state and inflammatory conditions?

What are the components of the HSPC inflammasome? Does ASC form specks upon activation of the HSPC inflammasome? Which kind of specks? Are these specks able to activate GSDMD?

What are the respective contributions of $\mathrm{IL}-1 \beta / \mathrm{IL}-18$ release, pyroptotic cell death, and GATA1 cleavage to HSPC and erythroid precursor differentiation?

Besides IL-1 1 , IL-18, GSDMD, and GATA1, are other proteins cleaved by the inflammasome in HSPCs in steady state and inflammatory conditions?

Since activated macrophages can release specks that activate bystander cells, are these specks able to reach the $\mathrm{BM}$ and modulate the activation of the HSPC inflammasome in inflammatory conditions?

In what ways can zebrafish models significantly complement other animal models to further elucidate many of these questions? 
15. Whyatt, D. et al. (2000) An intrinsic but cell-nonautonomous defect in GATA-1-overexpressing mouse erythroid cells. Nature 406, 519-524

16. Ferreira, R. et al. (2005) GATA1 function, a paradigm for transcription factors in hematopoiesis. Mol. Cell. Biol. 25, 1215-1227

17. Elias, H.K. et al. (2017) Molecular mechanisms underlying lineage bias in aging hematopoiesis. Semin. Hematol. 54, 4-11

18. Lamkanfi, M. and Dixit, V.M. (2014) Mechanisms and functions of inflammasomes. Cell 157, 1013-1022

19. Pilla, D.M. et al. (2014) Guanylate binding proteins promote caspase-11-dependent pyroptosis in response to cytoplasmic LPS. Proc. Natl. Acad. Sci. U. S. A. 111, 6046-6051

20. Santos, J.C. et al. (2018) LPS targets host guanylate-binding proteins to the bacterial outer membrane for non-canonical inflammasome activation. EMBO J. 37, e98089

21. Tyrkalska, S.D. et al. (2016) Neutrophils mediate Salmonella Typhimurium clearance through the GBP4 inflammasomedependent production of prostaglandins. Nat. Commun. 7 12077

22. Zwack, E.E. et al. (2017) Guanylate binding proteins regulate inflammasome activation in response to hyperinjected yersinia translocon components. Infect. Immun. 85, e00778-e00816

23. Sharma, D. and Kanneganti, T.D. (2016) The cell biology of inflammasomes: mechanisms of inflammasome activation and regulation. J. Cell Biol. 213, 617-629

24. Boucher, D. et al. (2018) Caspase-1 self-cleavage is an intrinsic mechanism to terminate inflammasome activity. J. Exp. Med. $215,827-840$

25. Martinon, F. et al. (2002) The inflammasome: a molecular platform triggering activation of inflammatory caspases and processing of proll-beta. Mol. Cell 10, 417-426

26. Guo, H. et al. (2015) Inflammasomes: mechanism of action, role in disease, and therapeutics. Nat. Med. 21, 677-687

27. Kayagaki, N. et al. (2015) Caspase-11 cleaves gasdermin D for non-canonical inflammasome signalling. Nature 526, 666-671

28. Shi, J. et al. (2015) Cleavage of GSDMD by inflammatory caspases determines pyroptotic cell death. Nature 526, $660-665$

29. Rathinam, V.A. and Fitzgerald, K.A. (2016) Inflammasome complexes: emerging mechanisms and effector functions. Cell 165 792-800

30. Tyrkalska, S.D. et al. (2019) Inflammasome regulates hematopoiesis through cleavage of the master erythroid transcription factor GATA1. Immunity 51, 50-63 e5

31. Masters, S.L. et al. (2012) NLRP1 inflammasome activation induces pyroptosis of hematopoietic progenitor cells. Immunity 37, 1009-1023

32. Ratajczak, M.Z. et al. (2019) NLRP3 inflammasome couples purinergic signaling with activation of the complement cascade for the optimal release of cells from bone marrow. Leukemia 33, 815-825

33. Lenkiewicz, A.M. et al. (2019) The NIrp3 Inflammasome orchestrates mobilization of bone marrow-residing stem cells into peripheral blood. Stem Cell Rev. Rep. 15, 391-403

34. Adamiak, M. et al. (2020) Nlrp3 inflammasome signaling regulates the homing and engraftment of hematopoietic stem cells (HSPCs) by enhancing incorporation of CXCR4 receptor into membrane lipid rafts. Stem Cell Rev. Rep. 16, 954-967

35. Basiorka, A.A. et al. (2016) The NLRP3 inflammasome functions as a driver of the myelodysplastic syndrome phenotype. Blood 128, 2960-2975

36. Mestas, J. and Hughes, C.C. (2004) Of mice and not men: differences between mouse and human immunology. J. Immunol. $172,2731-2738$

37. Shi, C. and Pamer, E.G. (2011) Monocyte recruitment during infection and inflammation. Nat. Rev. Immunol. 11, 762-774

38. Gunzer, M. (2014) Traps and hyper inflammation - new ways that neutrophils promote or hinder survival. Br. J. Haematol. 164, 189-199

39. Kolaczkowska, E and Kubes, P. (2013) Neutrophil recruitment and function in health and inflammation. Nat. Rev. Immunol. 13, 159-175

40. Martínez-Navarro, F.J. et al. (2020) Hydrogen peroxide in neutrophil inflammation: lesson from the zebrafish. Dev. Comp. Immunol. 105 103583
41. Köhler, A. et al. (2011) G-CSF-mediated thrombopoietin release 437 triggers neutrophil motility and mobilization from bone marrow 438 via induction of Cxcr2 ligands. Blood 117, 4349-4357 439

42. Bakele, M. et al. (2014) Localization and functionality of the 440 inflammasome in neutrophils. J. Biol. Chem. 289, 5320-5329 441

43. Yi, Y.S. (2018) Regulatory roles of the caspase-11 non-canonical 442 inflammasome in inflammatory diseases. Immune Netw. 18, e41 443

44. Schroder, K. and Tschopp, J. (2010) The inflammasomes. Cell 444 140, 821-832 445

45. Chen, K.W. et al. (2014) The neutrophil NLRC4 inflammasome 446 selectively promotes IL-1 beta maturation without pyroptosis 447 during acute Salmonella challenge. Cell Rep. 8, 570-582 448

46. Mariathasan, S. et al. (2004) Differential activation of the 449 inflammasome by caspase- 1 adaptors ASC and Ipaf. Nature 450 430, 213-218

47. Miao, E.A et al (2008) Pseudomonas aeruginosa activates 452 caspase 1 through Ipaf. Proc. Natl. Acad. Sci. U.S.A. 105, 453 2562-2567

48. Broz, P et al (2010) Differential requirement for Caspase-1 455 autoproteolysis in pathogen-induced cell death and cytokine 456 processing. Cell Host Microbe 8, 471-483 457

49. Chen, K.W. et al. (2016) The murine neutrophil NLRP3 458 inflammasome is activated by soluble but not particulate or crys- 459 talline agonists. Eur. J. Immunol. 46, 1004-1010 460

50. Pierini, R. et al. (2012) AIM2/ASC triggers caspase-8-dependent 461 apoptosis in Francisella-infected caspase-1-deficient macro- 462 phages. Cell Death Differ. 19, 1709-1721 463

51. Sagulenko, V. et al. (2013) AIM2 and NLRP3 inflammasomes ac- 464 tivate both apoptotic and pyroptotic death pathways via ASC. 465 Cell Death Differ. 20, 1149-1160 466

52. Chen, K.W. et al. (2018) Cutting edge: blockade of inhibitor of 467 apoptosis proteins sensitizes neutrophils to TNF- but not 468 lipopolysaccharide-mediated cell death and IL-1beta secretion. 469 J. Immunol. 200, 3341-3346

53. Frame, J.M. et al. (2020) Metabolic regulation of inflammasome 471 activity controls embryonic hematopoietic stem and progenitor 472 cell production. Dev Cell. Published online August 17, 2020. 473 https://doi.org/10.1016/j.devcel.2020.07.015 474

54. The International FMF Consortium (1997) Ancient missense mu- Q11 tations in a new member of the RoRet gene family are likely to 476 cause familial Mediterranean fever. Cell 90, 797-807 477

55. Van Opdenbosch, N. and Lamkanfi, M. (2019) Caspases in cell 478 death, inflammation, and disease. Immunity 50, 1352-1364 479

56. consortium, F.F (1997) A candidate gene for familial Mediterranean 480 fever. Nat. Genet. 17, 25-31 481

57. Hoffman, H.M. et al. (2001) Mutation of a new gene encoding a 482 putative pyrin-like protein causes familial cold autoinflammatory $\quad 483$ syndrome and Muckle-Wells syndrome. Nat. Genet. 29, 301-305 484

58. Hoffman, H.M. et al. (2001) Familial cold autoinflammatory 485 syndrome: phenotype and genotype of an autosomal dominant 486 periodic fever. J. Allergy Clin. Immunol. 108, 615-620 487

59. Zhong, F.L. et al. (2016) Germline NLRP1 mutations cause skin 488 inflammatory and cancer susceptibility syndromes via 489 inflammasome activation. Cell 167, 187-202 e17 490

60. Baroja-Mazo, A. et al. (2014) The NLRP3 inflammasome is re- 491 leased as a particulate danger signal that amplifies the inflamma- 492 tory response. Nat. Immunol. 15, 738-748 493

61. Franklin, B.S. et al. (2014) The adaptor ASC has extracellular and 494 'prionoid' activities that propagate inflammation. Nat. Immunol. 495 15, 727-737 496

62. Döring, Y. et al. (2017) Neutrophil extracellular traps in athero- 497 sclerosis and atherothrombosis. Circ. Res. 120, 736-743 498

63. Naruko, T. et al. (2002) Neutrophil infiltration of culprit lesions in 499 acute coronary syndromes. Circulation 106, 2894-2900 500

64. Chen, K.W. et al. (2018) Noncanonical inflammasome signaling 501 elicits gasdermin D-dependent neutrophil extracellular traps. 502 Sci. Immunol. 3, eaar6676 503

65. Sollberger, G. et al. (2018) Gasdermin D plays a vital role in the 504 generation of neutrophil extracellular traps. Sci. Immunol. 3, 505 eaar6689 506

66. Santoriello, C. and Zon, L.I. (2012) Hooked! Modeling human 507 disease in zebrafish. J. Clin. Invest. 122, 2337-2343 508

67. Howe, K. et al. (2013) The zebrafish reference genome se- 509 quence and its relationship to the human genome. Nature 496, 510 498-503 
68. Ellett, F. and Lieschke, G.J. (2010) Zebrafish as a model for vertebrate hematopoiesis. Curr. Opin. Pharmacol. 10, 563-570

69. Berman, J. et al. (2012) The zebrafish as a tool to study hematopoiesis, human blood diseases, and immune function. Adv. Hematol. 2012, 425345

70. Yoshida, N. et al. (2017) Macrophage-microbe interactions: lessons from the zebrafish model. Front. Immunol. 8, 1703

71. Renshaw, S.A. and Trede, N.S. (2012) A model 450 million years in the making: zebrafish and vertebrate immunity. Dis. Model. Mech. 5, 38-47

72. Meijer, A.H. (2016) Protection and pathology in TB: learning from the zebrafish model. Semin. Immunopathol. 38, 261-273

73. Kuri, P. et al. (2017) Dynamics of in vivo ASC speck formation. J. Cell Biol. 216, 2891-2909

74. Meijer, A.H. and Spaink, H.P. (2011) Host-pathogen interactions made transparent with the zebrafish model. Curr. Drug Targets 12, 1000-1017

75. Renshaw, S.A. et al. (2006) A transgenic zebrafish model of neutrophilic inflammation. Blood 108, 3976-3978

76. Hall, C. et al. (2007) The zebrafish lysozyme C promoter drives myeloid-specific expression in transgenic fish. BMC Dev. Biol. 7,42

77. Ellett, F. et al. (2011) mpeg1 promoter transgenes direct macrophage-lineage expression in zebrafish. Blood 117, e49-e56

78. Ganis, J.J. et al. (2012) Zebrafish globin switching occurs in two developmental stages and is controlled by the LCR. Dev. Biol. 366, 185-194

79. Traver, D. et al. (2003) Transplantation and in vivo imaging of multilineage engraftment in zebrafish bloodless mutants. Nat. Immunol. 4, 1238-1246
80. Tamplin, O.J. et al. (2015) Hematopoietic stem cell arrival 543 triggers dynamic remodeling of the perivascular niche. Cell 544 160, 241-252

81. De Maria, R. et al. (1999) Negative regulation of erythropoiesis by 546 caspase-mediated cleavage of GATA-1. Nature 401, 489-493 547

82. Ribeil, J.A. et al. (2007) Hsp70 regulates erythropoiesis by 548 preventing caspase-3-mediated cleavage of GATA-1. Nature 549 445, 102-105 550

83. Marzano, A.V. et al. (2018) A comprehensive review of neutro- 551 philic diseases. Clin. Rev. Allergy Immunol. 54, 114-130 552

84. Carney, T.J. et al. (2007) Inactivation of serine protease 553 Matriptase 1 a by its inhibitor Hai1 is required for epithelial integrity 554 of the zebrafish epidermis. Development 134, 3461-3471 555

85. Mathias, J.R. et al. (2007) Live imaging of chronic inflammation 556 caused by mutation of zebrafish Hai1. J. Cell Sci. 120, 3372-3383 557

86. Ludwig, L.S. et al. (2014) Altered translation of GATA1 in 558 Diamond-Blackfan anemia. Nat. Med. 20, 748-753 559

87. Danilova, N. et al. (2014) The role of the DNA damage response 560 in zebrafish and cellular models of Diamond Blackfan anemia 561 Dis. Model. Mech. 7, 895-905 562

88. Portincasa, P. (2016) Colchicine, biologic agents and more for 563 the treatment of familial Mediterranean fever. The old, the new, 564 and the rare. Curr. Med. Chem. 23, 60-86 565

89. Celkan, T. et al. (2005) The anemia of familial Mediterranean 566 fever disease. Pediatr. Hematol. Oncol. 22, 657-665 567

90. Prieur, A.M. et al. (1987) A chronic, infantile, neurological, cuta- 568 neous, and articular (CINCA) syndrome. A specific entity 569 analysed in 30 patients. Scand. J. Rheumatol. Suppl. 66, 57-68 570

91. Wu, W.C. et al. (2014) Circulating hematopoietic stem and pro- 571 genitor cells are myeloid-biased in cancer patients. Proc. Natl. 572 Acad. Sci. U. S. A. 111, 4221-4226 573 